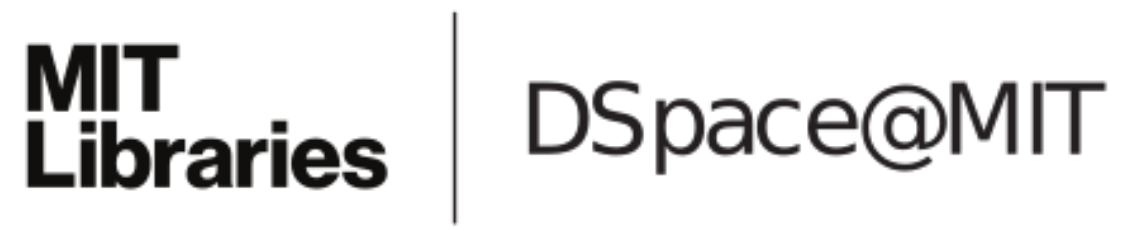

MIT Open Access Articles

Nanoscale strength distribution in
amorphous versus crystalline metals

The MIT Faculty has made this article openly available. Please share how this access benefits you. Your story matters.

Citation: Packard, C.E., O. Franke, E.R. Homer, and C.A. Schuh. "Nanoscale strength distribution in amorphous versus crystalline metals." Journal of Materials Research 25, no. 12 (December 31, 2010): 2251-2263. (C) 2010 Materials Research Society

As Published: http://dx.doi.org/10.1557/jmr.2010.0299

Publisher: Cambridge University Press (Materials Research Society)

Persistent URL: http://hdl.handle.net/1721.1/79783

Version: Final published version: final published article, as it appeared in a journal, conference proceedings, or other formally published context

Terms of Use: Article is made available in accordance with the publisher's policy and may be subject to US copyright law. Please refer to the publisher's site for terms of use. 


\title{
Nanoscale strength distribution in amorphous versus crystalline metals
}

\author{
C.E. Packard, O. Franke, E.R. Homer, and C.A. Schuh ${ }^{\text {a) }}$ \\ Department of Materials Science and Engineering, Massachusetts Institute of Technology, \\ Cambridge, Massachusetts 02139
}

(Received 9 July 2010; accepted 24 August 2010)

\begin{abstract}
Low-load nanoindentation can be used to assess not only the plastic yield point, but the distribution of yield points in a material. This paper reviews measurements of the socalled nanoscale strength distribution (NSD) on two classes of materials: crystals and metallic glasses. In each case, the yield point has a significant spread (10-50\% of the mean normalized stress), but the origins of the distribution are shown to be very different in the two materials classes. In crystalline materials the NSD can arise from thermal fluctuations and is attended by significant rate and temperature dependence. In metallic glasses well below their glass-transition temperature, the NSD is reflective of fluctuations in the sampled structure and is not very sensitive to rate or temperature. Computer simulations using shear transformation zone dynamics are used to separate the effects of thermal and structural fluctuations in metallic glasses, and support the latter as dominating the NSD of those materials at low temperatures. Finally, the role of the NSD as a window on structural changes due to annealing or prior deformation is discussed as a direction for future research on metallic glasses in particular.
\end{abstract}

\section{INTRODUCTION}

The advent of nanoindentation and other nanoscale mechanical tests has permitted the study of deformation mechanisms at the finest scales, often as fine as the carriers of plasticity themselves. For example, low-load spherical nanoindentation techniques have successfully detected subnanometer perturbations associated with "incipient plasticity" phenomena such as dislocation nucleation in crystalline materials ${ }^{1-7}$ and shear banding in metallic glasses. ${ }^{8-10}$ By applying low loads to impress a diamond tip into a test material while dynamically recording load and displacement with high resolution, deformation is stably promoted in a local region that is free from interference from prior sample deformation. What is more, due to the fine length scales of these tests, they can in principle be compared with atomistic or mechanistic simulations. ${ }^{11-14}$

A key strength of nanoindentation testing is that it permits rapid acquisition of data; hundreds of experiments can be conducted on a single sample in a matter of hours. This opens the door to new types of studies focused on statistics or spatial mapping of local mechan-

\footnotetext{
a) Address all correspondence to this author.

e-mail: schuh@mit.edu

This author was an editor of this journal during the review and decision stage. For the $J M R$ policy on review and publication of manuscripts authored by editors, please refer to http://www.mrs. org/jmr_policy

This paper has been accepted as an Invited Feature Paper.

DOI: $10.1557 / J M R .2010 .0299$
}

ical properties. For example, in composite microstructures an array of local nanoindentations can be used to map modulus, hardness, or other properties. ${ }^{15-20}$ Even within a single-phase sample, nanoindentation can be used to assess the statistical distribution of plastic events that may happen under nominally identical test conditions. Such measurement statistics can be used to infer details of the controlling physics that underlie deformation. In our group's recent work, we have studied the distribution of plastic events in materials with both crystalline $^{4,21,22}$ and amorphous ${ }^{23,24}$ structures, and in each case have been able to discern interesting details about the onset of plastic flow.

The purpose of this paper is twofold. First, we provide an overview of our recent work using low-load nanoindentation to measure the nanoscale strength distribution (NSD) in crystalline materials, including the methods and results of some prior published works. ${ }^{4,21-24}$ Second, we present new data on the NSD of several amorphous metals, which offers an intriguing contrast to the results from crystalline materials. The origins of the NSD in the two classes of materials are compared and contrasted, and implications for plasticity of metallic glasses are discussed.

\section{MEASURING THE NANOSCALE STRENGTH DISTRIBUTION}

For nanoindentation at low loads, it is possible to observe the elastic-plastic transition. In many materials the yield point is manifested as a "pop-in" event, which is 
characterized by a rapid depth excursion. Figure 1 shows some characteristic examples of load-displacement traces for (a) single-crystal (110) platinum and (b)-(d) three metallic glasses of different compositions, with the first pop-in event that marks the onset of global plasticity identified. In each of these four cases, the data were acquired with a nominally spherical diamond indenter tip, with further experimental details assembled in the Appendix.

In such experiments there are often many displacement bursts, so the identification of the yield point typically involves a more nuanced search for the first plastic event. For example, in each of the cases in Fig. 1, the first pop-in is also found to mark the first clearly measurable departure from the Hertzian elastic curve for sphere-on-plate contact ${ }^{25,26}$ :

$$
P=\frac{4}{3} E_{\mathrm{r}} R^{1 / 2} h^{3 / 2}
$$

where $P$ is the load, $R$ is the tip radius, $h$ is the indentation depth, and $E_{\mathrm{r}}$ is the reduced elastic modulus. The curve described by Eq. (1) is shown in each panel of Fig. 1 as a solid gray line (see the Appendix for experimental inputs). In addition to the pop-in in the loaddisplacement curves, for each of the four cases in Fig. 1, there was always a corresponding spike in indenter
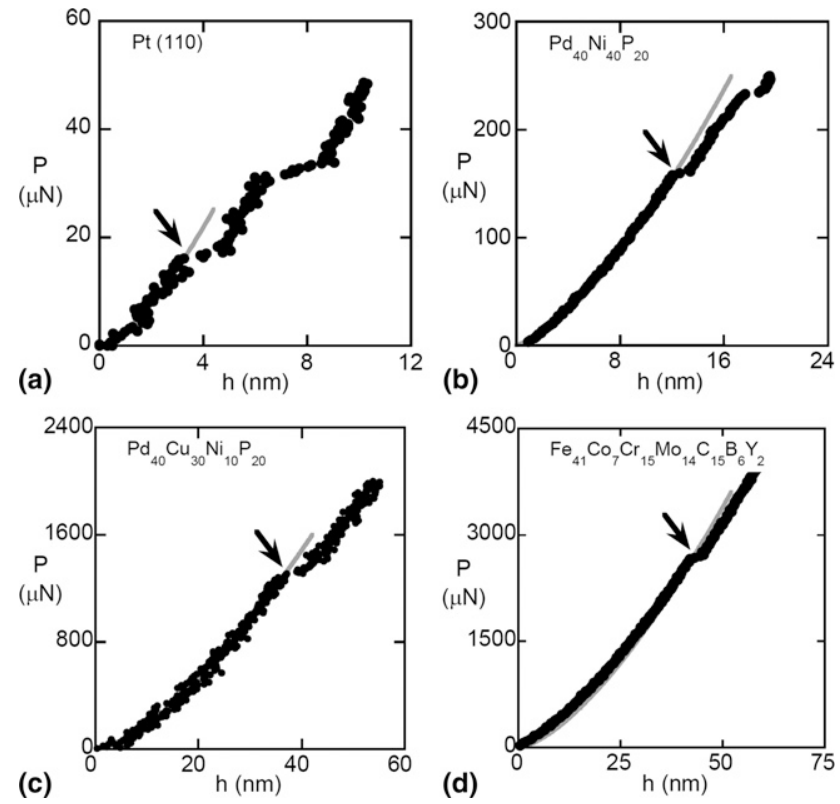

FIG. 1. Representative load-displacement curves that show the initial yield points of four materials, including (a) single-crystal (110) platinum, after Ref. 22, (b) $\mathrm{Pd}_{40} \mathrm{Ni}_{40} \mathrm{P}_{20}$ metallic glass, after Ref. 9, (c) $\mathrm{Pd}_{40} \mathrm{Cu}_{30} \mathrm{Ni}_{10} \mathrm{P}_{20}$ metallic glass, and (d) $\mathrm{Fe}_{41} \mathrm{Co}_{7} \mathrm{Cr}_{15} \mathrm{Mo}_{14} \mathrm{C}_{15} \mathrm{~B}_{6} \mathrm{Y}_{2}$ metallic glass, after Ref. 23. In each case, the experimental data are shown by black data points and compared to the predictions of the Hertz contact theory [Eq. (1)] for sphere-on-plate contact with the reduced moduli and indenter tip radii assembled in the Appendix. The first pop-in event is denoted by an arrow in each case. velocity, the analysis of which is elaborated in Refs. 4 and 9. The simultaneous observation of a displacement burst, velocity spike, and the first departure from elastic contact theory together are required to identify the yield point in the experiments reported here.

This paper is concerned not with the details of individual experiments such as those in Fig. 1, per se, but rather with the distribution of responses from many experiments. We assess the so-called nanoscale strength distribution (NSD) by repeating the experiment of Fig. 1 many times; we find that more than about 100 indentations are necessary to develop a clear picture of the distribution. These experiments are all conducted in the same general region of the same specimen at about the same time (within a few hours, generally), separated by a small distance that is sufficiently larger than the impression depth to guarantee that there is no overlap of the plastic zones. For a single spectrum, the same loading function (rates/times) is used for each experiment in the series; the experiments in the series are all nominally identical. The Appendix contains details for the four materials tested here.

Typical nanoscale strength distributions for each of the four materials in Fig. 1 are presented in Fig. 2. We
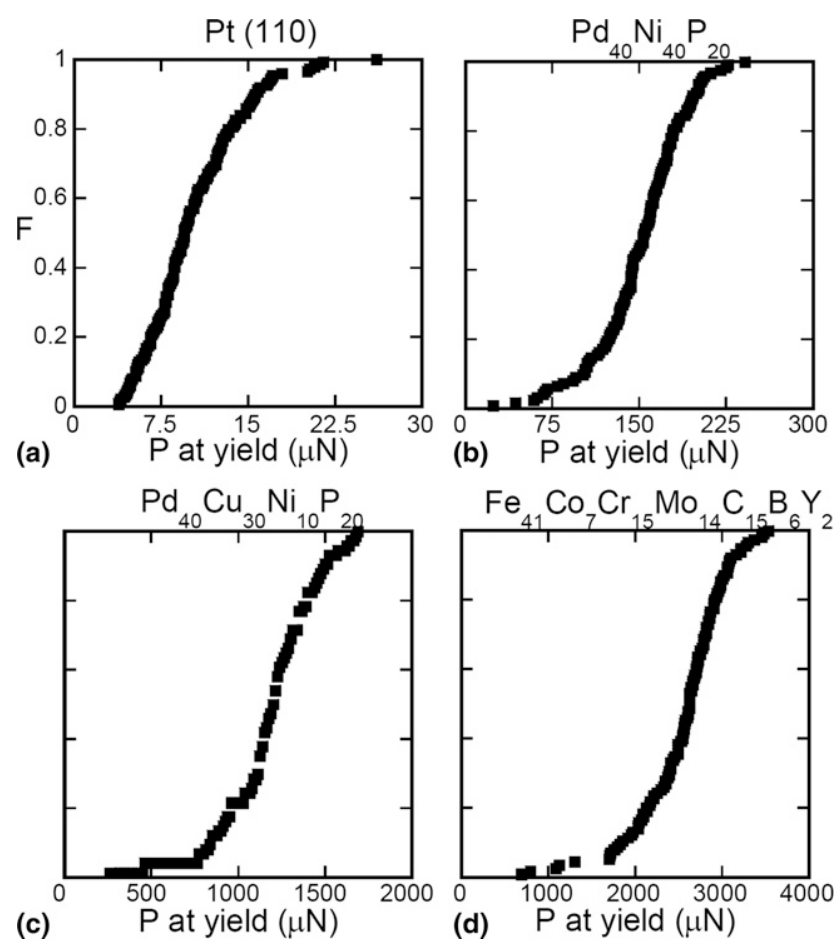

FIG. 2. Cumulative probability distributions for the load at the yield point or nanoscale strength distributions (NSDs) for the four experimental materials measured at room temperature: (a) single-crystal (110) platinum, (b) $\mathrm{Pd}_{40} \mathrm{Ni}_{40} \mathrm{P}_{20}$ metallic glass, (c) $\mathrm{Pd}_{40} \mathrm{Cu}_{30} \mathrm{Ni}_{10} \mathrm{P}_{20}$ metallic glass, and (d) $\mathrm{Fe}_{41} \mathrm{Co}_{7} \mathrm{Cr}_{15} \mathrm{Mo}_{14} \mathrm{C}_{15} \mathrm{~B}_{6} \mathrm{Y}_{2}$ metallic glass. The data in (a) appeared previously in Refs. 4 and 22, while that in (d) appeared previously in Refs. 23 and 24. The loading rates for these experiments were (a) 25 , (b) 0.2 , (c) 0.5 , and (d) $2.5 \mathrm{mN} / \mathrm{s}$. 
present the distributions by ordering the results to represent the cumulative fraction $(F)$ of yield loads for each material. A cumulative plot is preferred to a histogram, as the former permits every individual test to be represented by a single data point, while a histogram involves an arbitrary process of binning that can lead to biases in representation. Plotting the full distribution in this way reveals subtle changes to the distribution that might be obscured by limited sampling or by recording only average values. For all four materials in Fig. 2, the range of yield loads measured here significantly exceeds the measurement uncertainty in nanoindentation; the spread in the data are reflective of a true physical response, the origin of which is explored in more detail in subsequent sections.

The results in Fig. 2 are raw data, so comparison among different materials, or in some cases with physical models, requires normalization of the data in some way. For example, instead of the load at yield, the distribution could be presented in terms of a characteristic stress, such as the maximum (Hertzian) shear stress or the mean contact pressure at yield. To account for different moduli of the tested materials, a characteristic strain can also be used for normalization, such as, e.g., the mean contact pressure $\left(P_{\mathrm{m}}\right)$ divided by the reduced indentation modulus:

$$
\frac{P_{\mathrm{m}}}{E_{\mathrm{r}}}=\frac{4}{3 \pi} \sqrt{\frac{h}{R}}
$$

which we refer to for convenience as the "contact strain." Figure 3 replots all of the raw data from Fig. 2 using this parameter; the curves for all four materials now share the same scale.

In Figs. 1 to 3, two different classes of material (crystal versus glass) are presented, and the basic features of the elastic-plastic transition appear similar: the yield point manifests as a displacement burst that marks the departure from Hertzian contact theory (Fig. 1) and exhibits a broad statistical distribution (Fig. 3) that spans a similar range of contact strains. However, the process of plastic yield in crystalline and amorphous metals is, of course, physically very different. We can imagine two different sources for the spread in the NSDs in Fig. 3:

(i) Structural fluctuations: Across the many experiments used to collect the NSD, the indenter may sample many different atomic configurations (including both internal and surface configurations), each of which yields through a somewhat different critical event.

(ii) Thermal fluctuations: The same critical plastic event may be triggered upon each indentation, but it may be subject to stochastic noise from thermal fluctuations.

Of course, combinations of (i) and (ii) are also possible. In Secs. III and IV, we examine in more detail the NSDs of

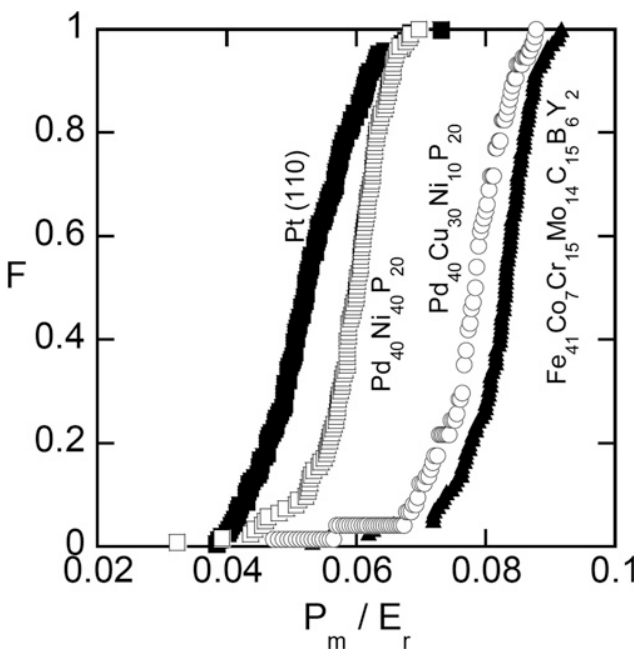

FIG. 3. The same nanoscale strength distributions from Fig. 2, normalized for geometry and elasticity: $P_{\mathrm{m}}$ is the mean contact pressure given by Eq. (2), and $E_{\mathrm{r}}$ is the reduced modulus of the contact (given in the Appendix for each material).

crystalline and amorphous materials, and demonstrate that their origins are dominated by (ii) and (i), respectively.

\section{NSD IN CRYSTALLINE MATERIALS}

We first consider experiments such as those conducted on (110) platinum in Figs. 1(a) and 2(a), in which the test material is a single crystal with an exposed surface plane that is the same for all of the indentations in the series. If a crystal is well annealed, and if its surface has been carefully prepared to render surface ledges or irregularities rare, then the initial defect density is low, and given the small size of the indentations, it is a fair assumption that all of the experiments are literally identical (to within an atomic translation). When such a homogeneous material is tested many times under identical conditions and widely variable results are obtained, one unavoidable source of variability - thermal fluctuation-is likely responsible. In this view, plasticity is controlled by a stress-biased, thermally activated process requiring some time for a suitable thermal fluctuation to occur and trigger it. As such, the process is variable and also very sensitive to time and temperature. Low temperatures reduce thermal fluctuations, and high imposed strain rates limit the available time for them to occur, both of which delay the yield event.

Thus, a critical piece of evidence for a thermal fluctuation origin of the NSD is its dependence on applied indentation rate and temperature. Figure 4(a) shows data from Ref. 4 for the (110) platinum specimen, where a shift in the NSD toward higher strength is seen as the loading rate is increased. Further evidence of thermal activation is seen in Fig. 4(b), which shows the effect of test temperature on the distribution, with higher temperatures promoting earlier yield. These two sets of data are 
representative of many other combinations of rate and temperature from Ref. 4, all of which follow the same basic trends and align with intuition for a thermally activated process.

Beyond the observation of rate- and temperaturedependent shifts to the NSD, it is possible to compare such data to the expectations of a thermal activation model. Schuh and Lund $^{21}$ originally laid out the framework for such analysis, which has been adapted and refined somewhat in subsequent works. ${ }^{4,22,27}$ Essentially, those authors assumed an Arrhenius rate law for the single, critical event that controls yield, incorporated a stress bias from the Hertzian elastic field that prevails beneath the indenter, and related the rate law to the probability of measuring the critical event during nanoindentation. The result is an estimate for the cumulative distribution function, which, in a simplified form for a condition of constant loading rate, writes ${ }^{4}$
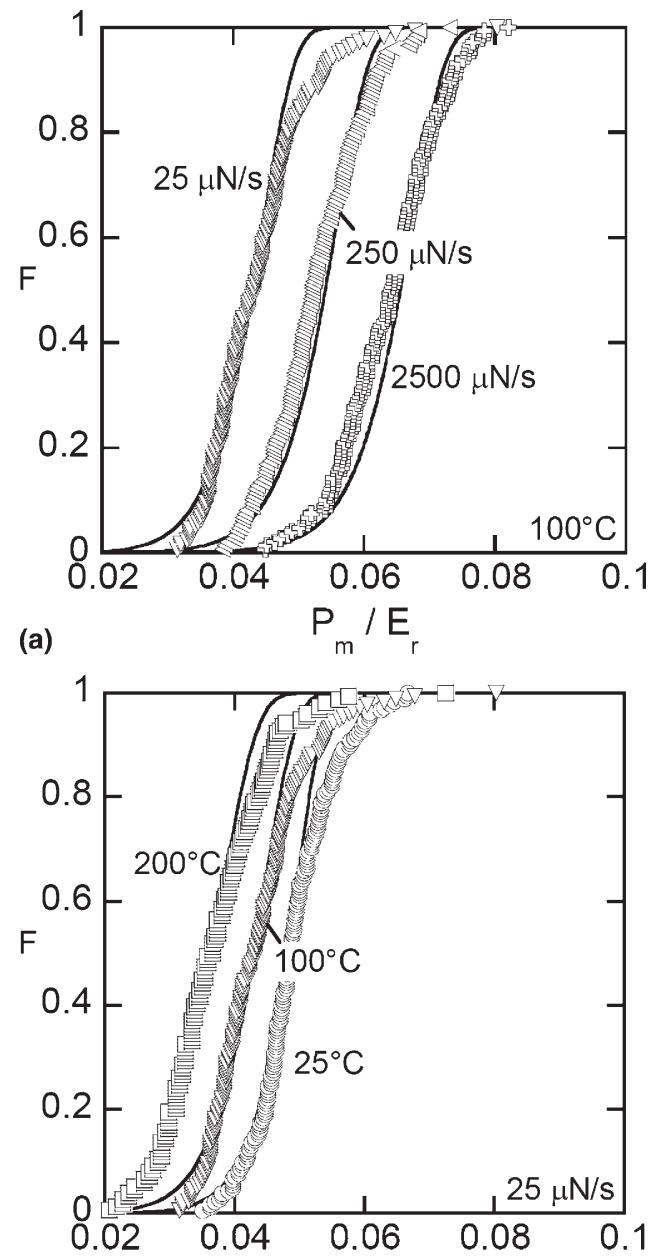

(b)

FIG. 4. Effect of (a) indentation rate and (b) test temperature on the NSD of single crystal (110) platinum. The solid lines show the simultaneous fit of all the data to a thermal activation model, of a form similar to that of Eq. (3). Adapted from Ref. 4.

$$
\begin{aligned}
F= & 1-\exp \left\{-\frac{9 \pi R \eta}{4 E_{\mathrm{r}} \dot{P} \alpha^{6}} \exp \left(-\frac{\varepsilon}{k T}\right)\left[120+\exp \left(P^{1 / 3} \alpha\right)\right.\right. \\
& \cdot\left(P^{5 / 3} \alpha^{5}-5 P^{4 / 3} \alpha^{4}+20 P \alpha^{3}-60 P^{2 / 3} \alpha^{2}+120 P^{1 / 3} \alpha\right. \\
& -120)]\},
\end{aligned}
$$

where $k$ is the Boltzmann constant and

$$
\alpha=\frac{0.47}{\pi}\left(\frac{4 E_{\mathrm{r}}}{3 R}\right)^{2 / 3} \frac{V}{k T} .
$$

Equation (3) contains both of the critical test parameters from Fig. 4, loading rate $\dot{P}$ and temperature $T$, and also contains the kinetic activation parameters for the process controlling yield: activation enthalpy $\varepsilon$, activation volume $V$, and attempt frequency $\eta$. A principal value of the model in Eq. (3) is that if, as hypothesized, a single critical process controls yield, then all of the NSD data measured on a single material can be fitted with this expression using one set of activation parameters. Not only does the model capture changes in the mean strength values with rate and temperature, it can in principle validate that all of the spread in the NSD itself arises from thermal fluctuations; for a set of identical experiments, some measurements sample favorable fluctuations that locally weaken the crystal, while others sample fluctuations that oppose plastic yield.

In the case of single-crystal Pt, this indeed turns out to be the case: a single thermally activated event with $\varepsilon \approx$ $0.34 \mathrm{eV}$ and $V \approx 10 \AA^{3}$ can essentially explain the full suite of experimental data (including the six NSD series in Fig. 4 as well as several others not shown). The solid curves shown in Fig. 4 show a fit of this kind [although with a more sophisticated model than the simplest form of Eq. (3) from Ref. 4], emphasizing that the shapes of the curves as well as their shifts with rate and temperature are all captured well with this kind of a model. NSDs extracted from a second crystal surface of Pt (111) found similar activation parameters, suggesting a similar ratelimiting mechanism for yield. ${ }^{4}$

Apart from Pt, other single crystalline materials have also been subjected to NSD analysis including $\mathrm{SiC}^{21}$ $\mathrm{Ta}^{28}$ and $\mathrm{Ni}_{3} \mathrm{Al}^{27}$; these always reveal a marked rate (and, where it is measured, temperature) dependence as expected for a thermally activated process. In every case where activation parameters have been extracted, they have been found to be very small-with scales characteristic of a single-atom process. The meaning of this has been discussed at length in Ref. 4; we believe that it generally suggests a heterogeneous dislocation nucleation process as underlying the first pop-in event, at least in the experiments from our group. Of course, in the various different materials under investigation the mechanism 
can certainly vary in many details, and a number of specific mechanisms remain under active discussion. ${ }^{22,28-32}$ One benefit of extracting activation parameters from a full NSD, though, is that these quantities are abstracted based on time, rate, and temperature dependencies; they are less sensitive to, e.g., the validity of Hertzian mechanics, nonlinear elastic effects, etc., than are estimates of the local stresses at yield and thus can provide more nuanced insight into mechanisms.

The fact that NSD data for single crystals are often extremely well fitted by a thermal activation model is interpreted as strong support for the notion that these types of experiments sample essentially the same critical event in each indentation, and the variability in the measured response is dominated by thermal fluctuations. The fact that the same amount of plastic strain is accommodated for every event in the distribution in some cases further supports this argument. ${ }^{21}$ However, such fits by no means rule out the possibility that additional variability in the NSD arises from structural fluctuations. Vacancies, impurities, surface ledges, pre-existing dislocations, etc., are all expected to occasionally be sampled during nanoindentation even at the finest scales. ${ }^{4,733}$ As pointed out in Ref. 4, these structural fluctuations introduce additional observation probabilities in the NSD, and could account for some features of the NSD that are not well explained by thermal activation. For example, it has been noted that the tails of the curves ${ }^{4}$ are usually the most poorly fitted by such models, and it seems reasonable that the outlier states of the distribution could arise from relatively rare structural fluctuations in single crystals.

Although the present work deals only with nanoindentation, the same statistical approach described here should be amenable to any nanoscale test geometry. For example, the discussion of yield and plasticity in microcompression testing is recently focusing attention on variability in results and the role of defect distributions. ${ }^{34}$ We suggest that study of the NSD and separation of thermal activation from structural fluctuations could provide quantitative insight on such issues.

\section{NSD IN AMORPHOUS METALS}

We now consider the NSD for amorphous metals such as shown in Figs. 2(b) to 2(d). Amorphous metals, or metallic glasses, have no lattice structure and do not deform by dislocation plasticity. Instead, the pop-ins observed during nanoindentation in metallic glasses correspond to rapid shear localization (shear banding) along a plane of material exceeding the yield stress of the bulk material. ${ }^{31,35}$ Flow serration in nanoindentation load-displacement curves is observed to be sensitive to composition, processing history, tip shape, applied loading rate, and temperature. ${ }^{31,36-44}$ The shear band corresponding to the initial yield event in low-load spherical indentation does not originate from the point of maximum shear stress in the Hertzian field (located at a distance of roughly half a contact radius into the material ${ }^{26,40}$ ), but instead is believed to span a trajectory ranging from the edge of the contact to a depth greater than twice the contact radius. ${ }^{9}$

Two considerations enter into our expectations about the NSD of metallic glasses. First, when a series of nanoindentations is conducted on a glass, although the test geometry and conditions are identical, the metallic glass lacks the chemical and structural homogeneity of crystalline material. There are a wide variety of topological states ${ }^{45-47}$ and free-volume sites ${ }^{48,49}$ located sporadically throughout the glass structure, and a corresponding spectrum of activation conditions to induce flow. ${ }^{50-54}$ Second, the process of shear localization that is believed to underlie the yield point is known to be a highly cooperative process. Although strain rate and temperature have a strong effect on the density and velocities of shear bands, ${ }^{55,56}$ the yield strength is characterized by a very large apparent activation volume and energy, and is therefore only weakly sensitive to temperature and rate. ${ }^{57}$ Based on these considerations, we expect that the large variability in the NSDs of the glasses in Figs. 2 and 3 is not principally related to thermal activation, but instead is predominantly a reflection of the structural variability of the glasses themselves. In the following, we proceed to verify this expectation through experiments that probe the rate and temperature dependence of the NSD, and then through simulations that model the unit process of glass deformation during nanoindentation.

\section{A. Temperature and strain rate effects}

As noted in Sec. III, a critical feature of thermal fluctuation-induced spread in the NSD is a corresponding significant dependence on rate and temperature. We now apply this test to some metallic glass specimens, by assessing rate sensitivity of the NSD over several orders of magnitude in indentation rate (for two glasses) and its temperature dependence below the glass transition (for a third glass). The Appendix contains all of the experimental details for these series of indentations.

We first turn our attention to the temperature experiments. Although our group ${ }^{55,58}$ and others ${ }^{44}$ have applied high-temperature nanoindentation to metallic glasses in the past, the present experiments are the first to explicitly study the first yield event at nonambient temperatures. We note that these experiments require a customized apparatus capable of maintaining a nonoxidizing environment, so that the measured results can be confirmed free of artifacts from surface oxide layers (see the Appendix). Figure 5 presents some examples of the raw loaddisplacement curves for these tests. All of the curves shown are for temperatures below the glass transition $\left(288{ }^{\circ} \mathrm{C}^{59}\right)$, so the basic response is the same, including 
apparent elastic deflection and a pop-in marking the onset of measurable plastic flow.

The NSD of this Pd-based glass is broad (see Fig. 3), and we find that temperature lowers the mean yield point of the glass. While at room temperature the median value of $P_{\mathrm{m}}$ at yield is $7.8 \mathrm{GPa}$, it drops by about $10 \%$ at $50{ }^{\circ} \mathrm{C}$ and another $5 \%$ at $100{ }^{\circ} \mathrm{C}$, to values of 7.0 and $6.6 \mathrm{GPa}$, respectively. This softening is relatively minor, and at least partly due to the lowering of the elastic modulus with temperature. When the NSDs are plotted using the modulus-compensated mean pressure (or characteristic strain) as in Fig. 6, the curves for these three temperatures are quite close together.

At a somewhat higher temperature of $150{ }^{\circ} \mathrm{C}$, which is about $75 \%$ of the glass transition temperature of this glass on the absolute scale, the measured behavior shows a deviation from expectations, with the NSD shifted to apparently higher strengths. As seen in Fig. 5, the loaddisplacement curves at this temperature have significantly lower curvature in the range below the nominal yield point. In fact, these curves are not well fitted by the Hertzian contact theory [Eq. (1)], which we believe is a reflection of time-dependent (viscous) flow mechanisms setting in; it is common to observe such mechanisms beginning to emerge at these temperatures for metallic glasses. ${ }^{55}$ Accordingly, the physics of flow are different at this temperature. The first pop-in measured is not necessarily reflective of the same physical event, and the Hertzian calculations used to analyze the data are no longer strictly valid. As a result, the NSD at this temperature lies notably above those for the lower temperatures in Fig. 6. Even so, the data clearly show that there is no strong temperature dependence of the NSD in this material. [Compared with the effect of temperature in single

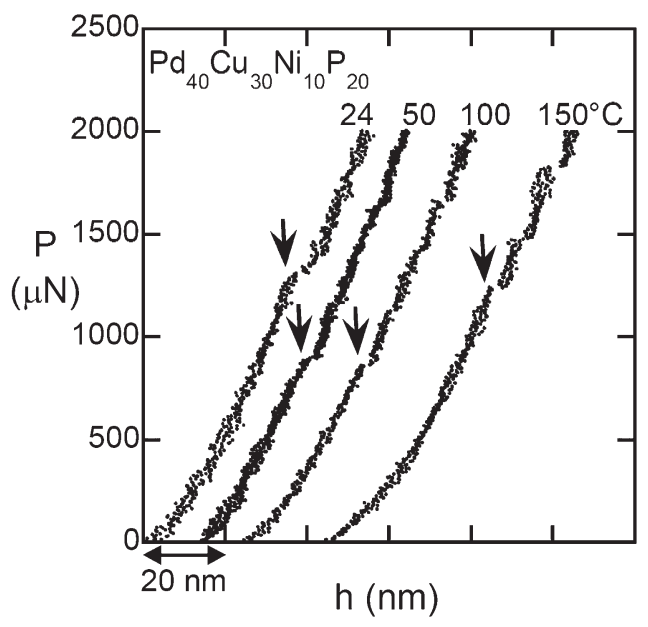

FIG. 5. Examples of the load-displacement curves measured on the $\mathrm{Pd}_{40} \mathrm{Cu}_{30} \mathrm{Ni}_{10} \mathrm{P}_{20}$ metallic glass at elevated temperatures. The curves are offset along the $\mathrm{x}$-axis for clarity of presentation, and arrows denote the specific location of the first pop-in event in these tests. crystal Pt, for example, in Fig. 4(b), the trend here is negligible.]

Next, turning our attention to the indentation rate experiments, we find that the effect of rate on the NSD curves is also negligible. Our data for two different glasses are shown in Fig. 7, where the various-rate data sets are tightly grouped and follow the same general shape, despite several decades of difference in the applied indentation rates.

The above results on the NSD of metallic glasses are in stark contrast to those for single crystal Pt in Fig. 4.

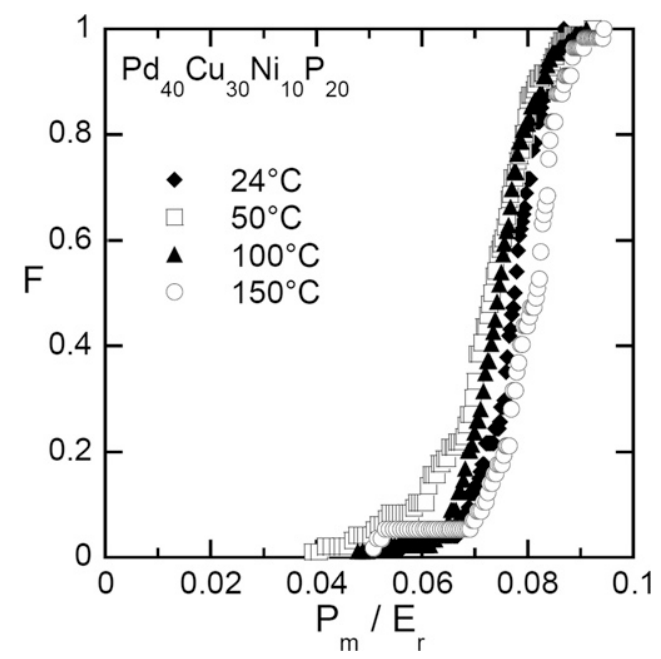

FIG. 6. Nanoscale strength distribution of $\mathrm{Pd}_{40} \mathrm{Cu}_{30} \mathrm{Ni}_{10} \mathrm{P}_{20}$ metallic glass, measured at four different temperatures. When normalized by temperature-dependent reduced modulus, the data for the lower temperatures agree reasonably well, suggesting weak temperature dependence of the specific plastic deformation mechanism that controls yield. The data at $150{ }^{\circ} \mathrm{C}$ are anomalously high because the temperature is approaching the range where mechanisms shift (see text).

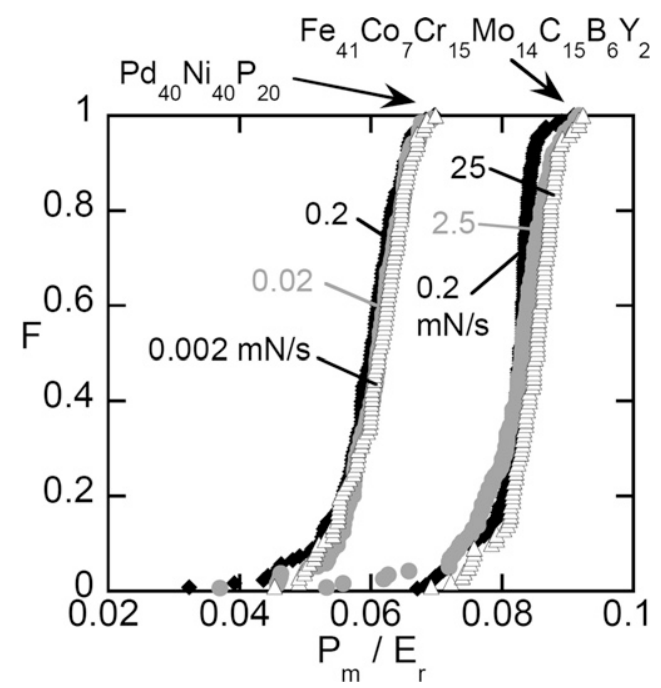

FIG. 7. Nanoscale strength distribution (NSD) of two metallic glasses is found to be essentially unchanged as the rate of the test is varied over two orders of magnitude. 
In Pt, the spread of the NSD as well as its change with rate and temperature are all consistent with thermal activation, whereas in metallic glasses there is a large spread in measured strength but essentially no rate dependence. The temperature dependence of the metallic glass NSD is also trivially compensated for by the temperature-dependent modulus, at least at low temperatures well below the onset of viscous creep flow.

In one sense, these results are exactly as expected for metallic glasses deformed at low temperatures where shear banding prevails: yield is known to be strain controlled and thus strength scales with modulus, ${ }^{50,57,60}$ while the essential rate-independence of flow is also well documented. ${ }^{57}$ The negligible temperature and rate dependence suggest that thermal activation plays a relatively smaller role in the yield of these materials (at least for temperatures well below the glass transition), and despite this, there is a very large spread in the NSD. We therefore conclude that in metallic glasses, the NSD spread does not principally arise from thermal fluctuations in the system. [Although one could attempt to fit any individual dataset from Figs. 6 or 7 with a thermal activation model like that of Eq. (3), it is not possible to fit all of the rate and temperature series with a single set of activation parameters.] Instead, we suggest that the NSD data represent an actual distribution of local strengths, controlled by the local atomic configuration sampled beneath the indenter tip.

\section{B. Computer simulations}

One way to verify the above inference-that structural fluctuations inherent to the glass structure cause the spread in the NSD data-is to use computer simulation to explicitly study the effects of structural heterogeneity on nanoindentation. Here we make use of a newly developed mesoscale simulation method for metallic glasses, termed "shear transformation zone dynamics."12,61,62 The shear transformation zone (STZ) is envisioned as the unit process of plastic deformation in metallic glasses and is essentially a local shear rearrangement of a cluster of atoms; through many such local shear transformations distributed through the glass volume, global plasticity of the glass is realized. The STZ dynamics modeling framework considers a coarse-grained representation of a metallic glass on a finite element mesh, where potential STZs are mapped onto features of the mesh and can be activated throughout the simulation cell according to a rate law that depends on the local stress and temperature. The framework is controlled by the kinetic Monte Carlo (KMC) algorithm, and the stress and strain distributions following the volume-preserving shear-shape distortions of STZs are calculated using finite element analysis (FEA). The combination of KMC and FEA provides the ability to capture the expected behaviors of a metallic glass on experimentally relevant time and length scales, while offering a microscopic picture of the deformation. ${ }^{12}$

In principle, the STZ dynamics model can closely match experimental length and time scales, and here we use a model that is somewhat smaller but reasonably comparable in scale to what is experimentally achievable in nanoindentation. Specifically, we use a twodimensional plane-strain model, which is indented by a cylindrical indenter of $40 \mathrm{~nm}$ radius, at a fixed rate of $1 \mathrm{~nm} / \mathrm{s}$. However, the purpose of the model is not to provide predictive comparisons with the experiments, but to separately study the effects of thermal and structural fluctuations. We achieve this separation as follows. The simulated glass structure that we study is prepared by simulated cooling from the liquid, from 1000 to $300 \mathrm{~K}$ at a rate of $10 \mathrm{~K} / \mathrm{s}$, as described in more detail in the Appendix. Such processing leads to considerable quenched-in disorder, in the form of a pre-existing distribution of local strains and stresses. The starting condition of the material is shown graphically in Fig. 8, where the local STZ strains are shaded in Fig. 8(a), and the local von Mises stresses are shown in Fig. 8(b). With the structure shown in Fig. 8, we conduct two different series of simulations:

(i) In the first series, 10 copies of the simulation cell are produced, and 10 simulated indentations in exactly the same location are conducted, but with different random number generator seeds. In this series, the simulations therefore sample the same structure but different thermal fluctuations.

(ii) In the second series, 10 variants of the simulation cell were produced, by translating and rotating it by $180^{\circ}$ (in various combinations) relative to the indenter

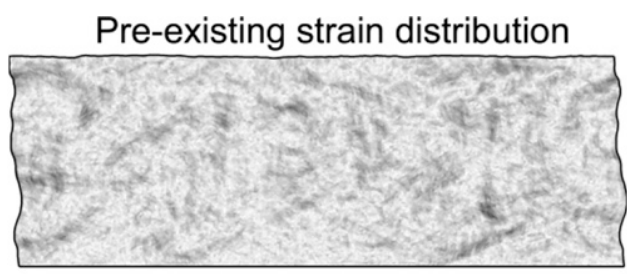

(a)

Pre-existing stress distribution

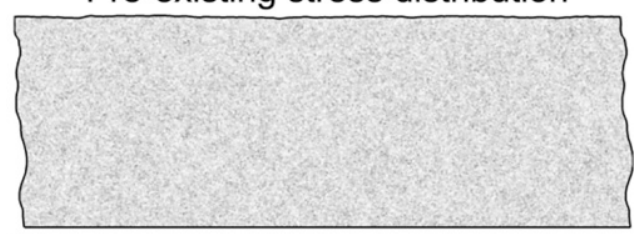

(b)

FIG. 8. Representations of the simulation cell used for the STZ dynamics simulation of indentation, where the local (a) total STZ strain and (b) von Mises stress distribution are colored in grayscale. These images illustrate the variety of local states contained in the simulated glass and which are sampled during a nanoscale contact. 
position. In this series, the indentations therefore sample 10 different local glass structures (as well as different thermal fluctuations).

The NSD analysis proceeds in essentially the same way for these simulations as it did for the experiments. An example raw load-displacement curve for one simulation is shown in Fig. 9, where it is compared with the elastic deflection curve. In these simulations the departure from elastic loading is not marked by a severe popin, for a number of reasons discussed elsewhere, ${ }^{23}$ including the size of the indenter and the lack of a strain softening mechanism in the simulation. However, the yield point can be easily identified by recording the very first STZ activation, with its corresponding triggering load, contact pressure, and indenter displacement (with a degree of resolution higher than is experimentally possible). Upon subsequent loading, additional STZs form and gradually develop into individual slip lines, as shown in the inset to Fig. 9 at a later time well into the plastic regime.

After conducting each series of indentations, the NSD is compiled as shown in Fig. 10, using the contact strain for the ordinate, as done for the experimental data previously. First examining the data labeled "series 1: thermal fluctuations," we directly see the role of thermal activation on the NSD, as these indentations all sampled exactly the same glass configuration. The degree of spread in the NSD in this case is small (9\% of the mean), and the curve is almost vertical with most of the variation located in the tails. On the other hand, for "series 2: structural fluctuations," the spread is significantly larger ( $20 \%$ of the mean), and the shape of the distribution is

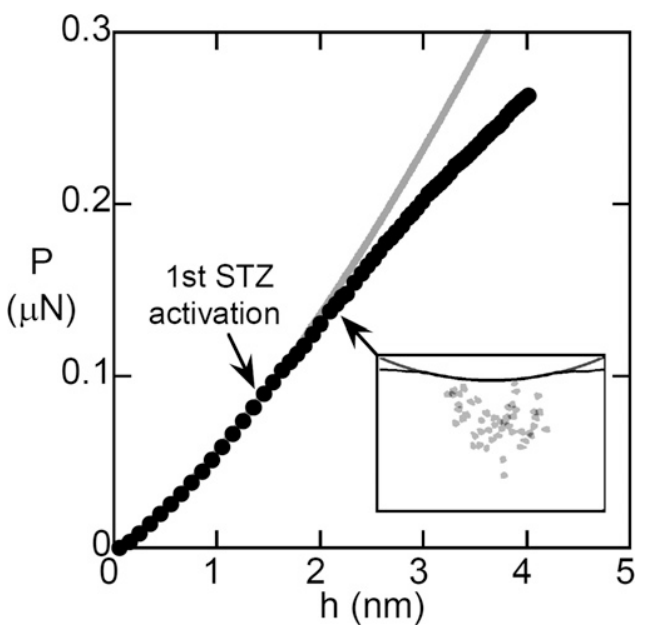

FIG. 9. Typical simulation result showing the load-displacement result for simulated nanoindentation of a metallic glass with a preexisting stress distribution. The data gradually depart from the elastic curve (solid gray line), as a plastic zone of STZ activity develops (see inset). The first plasticity to occur is difficult to discern in the displacement of the indenter, but is identifiable based on the activation of the first STZ, at the point noted. different, comprising a shallower slope across the full range.

Comparing the raw simulation results to the experiments is not strictly appropriate (as a result of the differences in geometry, glass properties, etc.). However, on a normalized basis some limited comparisons are possible. For example, it is significant that the spread in the experimental NSDs (between, say, the 10th and 90th percentile) is always close to $\sim 20 \%$ of the mean (see Fig. 3), similar to what is seen in the simulations that probe structural fluctuations, but significantly broader than seen in the simulations that sample only thermal fluctuations. In fact, for the glass that most closely matches the elastic and thermal properties of the simulated glass $\left(\mathrm{Pd}_{40} \mathrm{Cu}_{30} \mathrm{Ni}_{10} \mathrm{P}_{20}\right)$, the correspondence between the simulated NSD and the experimental one is encouraging, with the $(10,50,90)$ th percentile values of $P_{\mathrm{m}} / E_{\mathrm{R}}$ being $(0.068,0.075,0.084)$ and $(0.067,0.078,0.084)$, respectively. These results provide support for our interpretation that variability in the local glass structure is the dominant source of the spread in the experimental NSDs of metallic glasses.

\section{Induced structural variations}

Given that the spread in measured yield points derives from structural variations in metallic glasses, it follows that the NSD might be sensitive to changes in the glass structure. We offer this as a key direction for future studies of metallic glasses, since the connection between glass structure and properties is poorly understood and usually based only on macroscopic measurements that implicitly average over the NSD. We offer two examples of the kinds of studies that are needed in this area below.

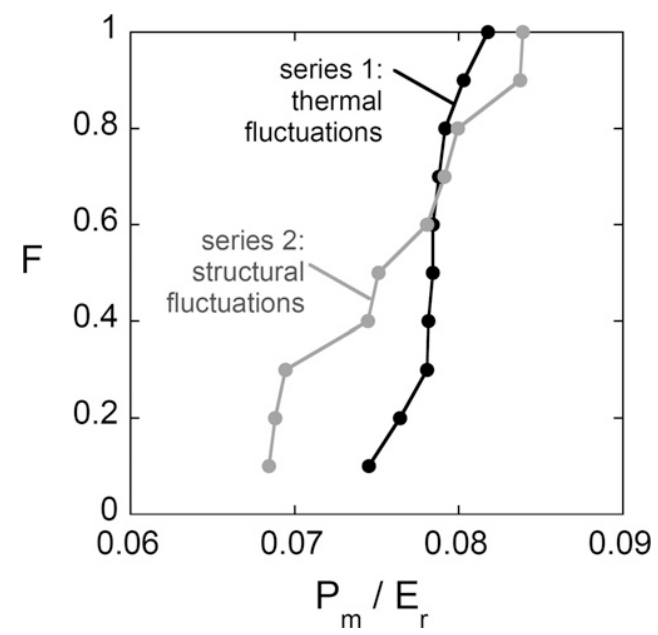

FIG. 10. Nanoscale strength distributions for the glass simulated by STZ dynamics. Here the first plasticity is measured as the point of the first STZ activation (see Fig. 9) for two sets of simulations with different fluctuations imposed. For series 1 , only thermal fluctuations are different among the tests in the series, while for series 2 , the structure under the indenter was different in each of the 10 cases. 
First, the most widely appreciated method of changing glass structure is to anneal and relax out free volume. ${ }^{57,63,64}$ In Fig. 11, we compare the NSD on two different glasses in as-cast and well-annealed states $(12 \mathrm{~h}$ at $80 \%$ of the glass-transition temperature). The effect of annealing is found to be extremely subtle: if only a few measurements were conducted then there would be no clear change in the average yield point or its standard deviation. Only by assembling a thorough cumulative NSD do we observe any change at all, and even then the results are still subtle (although consistent): there is a slight tendency toward strengthening at all points in the distribution for both of the glasses tested here. Perhaps more interesting is that the lower tail of the NSD seems most affected by annealing. This suggests that the "weakest" sites in the glass structure may be preferentially removed; if the weak sites are presumed to be those of the highest free volume, then the present results may offer an interesting comparison with observed changes in positron annihilation data that show the loss of high free-volume sites upon annealing. ${ }^{49}$ Correspondingly, the higher tail of the NSD is relatively less affected, which suggests that "strong" sites are not susceptible to relaxation. On the whole, though, the average strength of the glass is only slightly affected by annealing. This aligns well with macroscopic measurements, which generally show little change in strength or hardness after annealing. Annealing has a far more pronounced effect on toughness ${ }^{65,66}$ - this may relate to the preferential loss of "soft" sites in the NSD, although much more work is needed to make a true connection between these local measurements and any global property.

Second, glass structure may be affected by deformation, and in some of our recent work this has been observed during cyclic loading under the nanoindenter. ${ }^{23,24}$ Figure 12 shows data reproduced from those works,

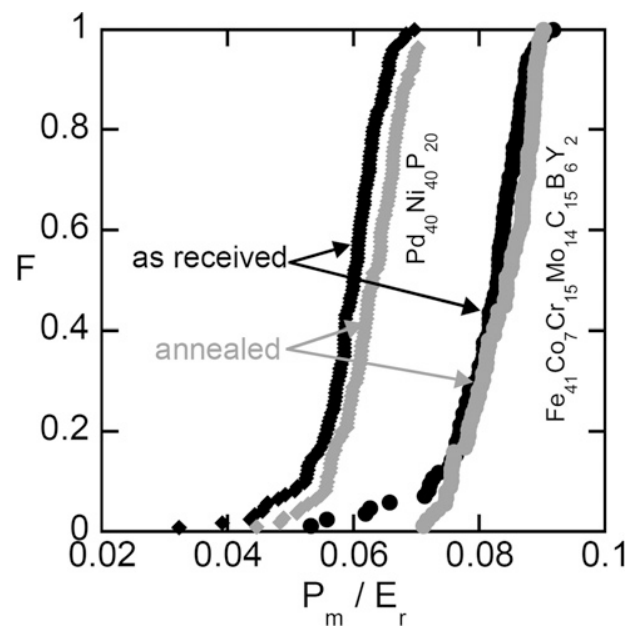

FIG. 11. Nanoscale strength statistics for metallic glasses in as-cast and annealed states. (a) $\mathrm{Pd}_{40} \mathrm{Ni}_{40} \mathrm{P}_{20}$ and (b) $\mathrm{Fe}_{41} \mathrm{Co}_{7} \mathrm{Cr}_{15} \mathrm{Mo}_{14} \mathrm{C}_{15} \mathrm{~B}_{6} \mathrm{Y}_{2}$. The annealing was conducted at $80 \%$ of the glass transition for $12 \mathrm{~h}$.
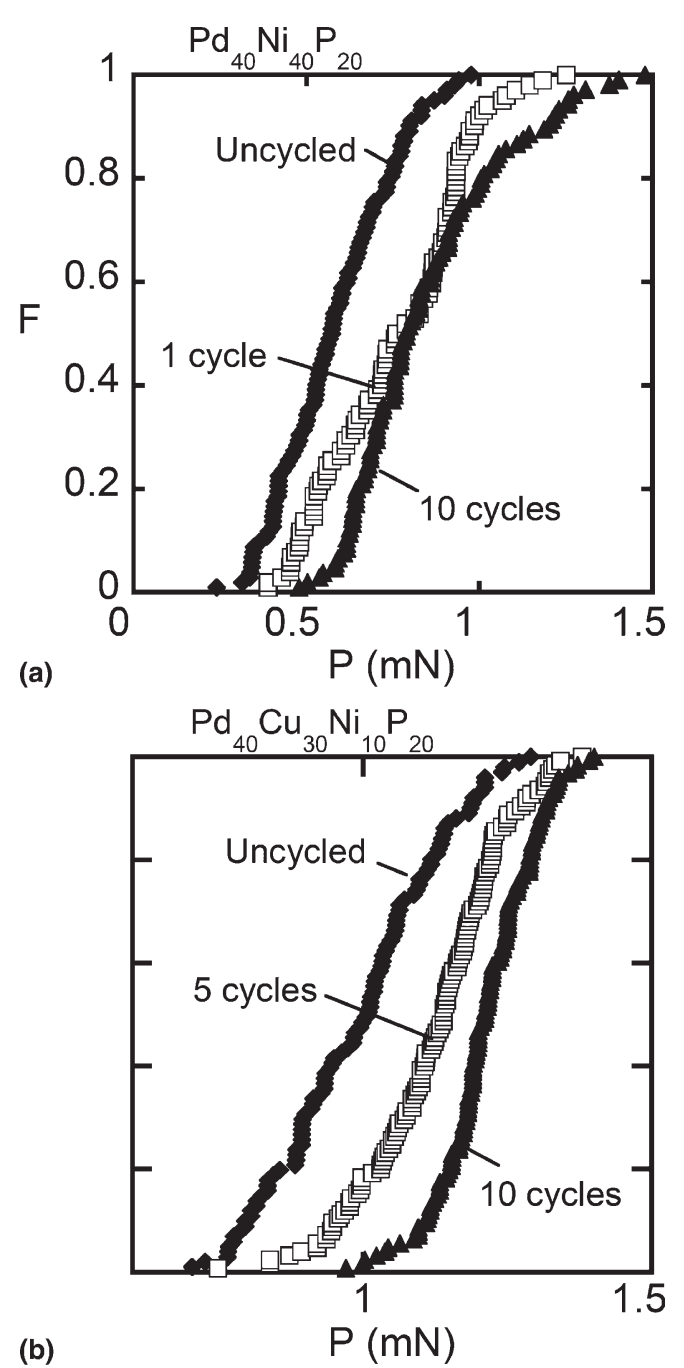

(b)

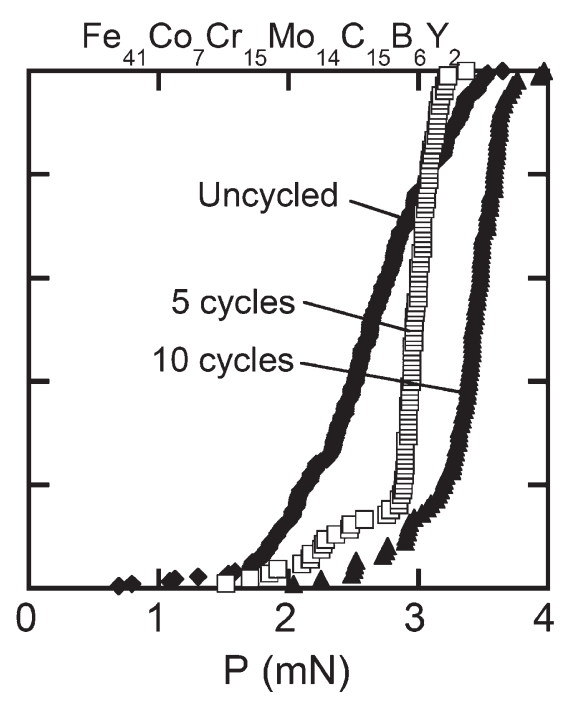

FIG. 12. Example data showing the shift in the NSD of metallic glasses subjected to prior cyclic loading in the nominal elastic range. In each of the three glasses, substantial cyclic hardening is observed. These data appeared previously in Refs. 23 and 24; note that the data for $\mathrm{Pd}_{40} \mathrm{Ni}_{40} \mathrm{P}_{20}$ here used a different tip radius $(1.1 \mu \mathrm{m})$ than the data in the rest of this paper. 
plotting the NSD both for the as-received glass and for glass that was subjected to sub-yield cycles of indentation (in the nominal Hertzian elastic regime) before being loaded up to the yield point. For all three glasses, the NSD is found to strongly shift to the right after cyclic loading, and in general, the effect is progressive over the course of a few cycles. This cyclic hardening phenomenon is clear (although indirect) evidence that cycles of nominal elastic loading, during which no shear bands form, lead to microplastic structural rearrangement of the glass. Interestingly, these deformation-induced structural changes far more drastically impact the NSD than does annealing (compare Figs. 11 and 12). In annealing, even a small effect seen in the NSD manifests as a large effect on, e.g., fracture toughness; the comparatively large effect of cyclic microplasticity could perhaps be expected to have an even more significant effect on macroscopic properties. The connections of this phenomenon to fatigue of metallic glasses are being explored presently, as a local mechanism for glass structure change is required to explain conventional cyclic crack advance during fatigue of metallic glasses. 57

\section{CONCLUSION}

We propose that the combination of nanoscale testing with large statistical datasets is a powerful approach to understanding deformation mechanisms. From a series of nominally identical nanoscale experiments, not only the mean measurement, but also its full statistical distribution, are reflective of details of the nanoscale fluctuations that control properties. Here we have reviewed measurements of the nanoscale strength distribution (NSD) for two classes of materials: crystals and amorphous metals. In each case, the NSD is assessed by low-load spherical nanoindentation measurements, and in each case a broad spread of results emerges. However, the details of these NSDs and their variation are distinctly different between crystals and glasses, which is a direct result of the fundamentally different nature of plastic flow in these classes of materials.

In crystalline metals, the spread of the NSD as well as its change with indentation rate and test temperature can all be essentially explained based on thermal activation. Thermal noise is the principal source of variability in such systems, because every test samples essentially an identical atomic environment. The yield event is a stressbiased dislocation-based process, and the activation parameters from experiments reported to date are generally consistent with a heterogeneous mechanism of dislocation multiplication or nucleation.

In metallic glasses well below their glass transition, the variability in the yield point is dominated not by thermal fluctuations, but by structural ones; nominally identical tests probe different local atomic structures, which intrinsically have different yield strengths. Here we have presented new experimental data that confirm this proposal. The effects of rate and temperature upon the NSD of metallic glasses are essentially negligible, which in turn means that thermal fluctuations cannot be the primary cause of the spread in the NSD. This inference has also been tested using an STZ dynamics model of nanoindentation in metallic glass, which permits a separation of thermal and structural fluctuations on the measured NSD that is not possible in experiment. These simulations confirm that in a glass below the glass transition, the structural variability probed during a series of nanoindentations is the more significant source of spread in the NSD than is thermal noise.

Finally, we observe that because the NSD of metallic glasses is reflective of their underlying distribution of structures, it can be thought of as a sensitive probe for local structural changes. Here we describe two specific processes of structural change: annealing and cyclic loading. Annealing is found to shift the NSD in a relatively subtle way; it does not change the average strength of the glass by much, but does seem to preferentially remove weaker regions of the glass. On the other hand, cyclic loading in the nominal elastic range (before a shear band forms) leads to local structural change that is attended by hardening.

\section{ACKNOWLEDGMENTS}

This work was supported by the United States Office of Naval Research under Contract No. N00014-0810312. The authors would like to thank Dr. Y. Li (National University of Singapore) and Dr. J. Shen (Harbin Institute of Technology) for providing the glasses used in this work. The experimental involvement of Christopher Wallace (MIT) is gratefully acknowledged. E.R.H. was supported through the National Defense Science and Engineering Graduate (NDSEG) fellowship.

\section{REFERENCES}

1. D.F. Bahr and G. Vasquez: Effect of solid solution impurities on dislocation nucleation during nanoindentation. J. Mater. Res. 20, 1947 (2005).

2. D.F. Bahr, D.E. Wilson, and D.A. Crowson: Energy considerations regarding yield points during indentation. J. Mater. Res. 14, 2269 (1999).

3. A.C. Lund, A.M. Hodge, and C.A. Schuh: Incipient plasticity during nanoindentation at elevated temperatures. Appl. Phys. Lett 85, 1362 (2004).

4. J.K. Mason, A.C. Lund, and C.A. Schuh: Determining the activation energy and volume for the onset of plasticity during nanoindentation. Phys. Rev. B 73, 054102 (2006).

5. T.A. Michalske and J.E. Houston: Dislocation nucleation at nanoscale mechanical contacts. Acta Mater. 46, 391 (1998).

6. S. Suresh, T.G. Nieh, and B.W. Choi: Nano-indentation of copper thin films on silicon substrates. Scr. Mater. 41, 951 (1999).

7. S.G. Corcoran, R.J. Colton, E.T. Lilleodden, and W.W. Gerberich: Anomalous plastic deformation at surfaces: Nanoindentation of gold single crystals. Phys. Rev. B 55, 16057 (1997). 
8. H. Bei, Z.P. Lu, and E.P. George: Theoretical strength and the onset of plasticity in bulk metallic glasses investigated by nanoindentation with a spherical indenter. Phys. Rev. Lett. 93, 125504 (2004)

9. C.E. Packard and C.A. Schuh: Initiation of shear bands near a stress concentration in metallic glass. Acta Mater. 55, 5348 (2007).

10. W.J. Wright, R. Saha, and W.D. Nix: Deformation mechanisms of the $\mathrm{Zr}_{40} \mathrm{Ti}_{14} \mathrm{Ni}_{10} \mathrm{Cu}_{12} \mathrm{Be}_{24}$ bulk metallic glass. Mater. Trans. 42, $642(2001)$

11. Y.F. Shi and M.L. Falk: Stress-induced structural transformation and shear banding during simulated nanoindentation of a metallic glass. Acta Mater. 55, 4317 (2007).

12. E.R. Homer and C.A. Schuh: Mesoscale modeling of amorphous metals by shear transformation zone dynamics. Acta Mater. 57, 2823 (2009).

13. J. Li, K.J. Van Vliet, T. Zhu, S. Yip, and S. Suresh: Atomistic mechanisms governing elastic limit and incipient plasticity in crystals. Nature 418, 307 (2002).

14. K.J. Van Vliet, J. Li, T. Zhu, S. Yip, and S. Suresh: Quantifying the early stages of plasticity through nanoscale experiments and simulations. Phys. Rev. B 67, 104105 (2003).

15. O. Franke, K. Durst, and M. Goken: Nanoindentation investigations to study solid solution hardening in Ni-based diffusion couples. J. Mater. Res. 24, 1127 (2009).

16. O. Franke, K. Durst, and M. Goken: Microstructure and local mechanical properties of Pt-modified nickel aluminides on nickel-base superalloys after thermo-mechanical fatigue. Mater. Sci. Eng., A 467, 15 (2007).

17. K. Tai, M. Dao, S. Suresh, A. Palazoglu, and C. Ortiz: Nanoscale heterogeneity promotes energy dissipation in bone. Nat. Mater. 6 , 454 (2007)

18. C.A. Tweedie, D.G. Anderson, R. Langer, and K.J. Van Vliet: Combinatorial material mechanics: High-throughput polymer synthesis and nanomechanical screening. Adv. Mater. 17, 2599 (2005).

19. G. Constantinides, K.S.R. Chandran, F.J. Ulm, and K.J. Van Vliet: Grid indentation analysis of composite microstructure and mechanics: Principles and validation. Mater. Sci. Eng., A 430, 189 (2006).

20. J.C. Zhao, M.R. Jackson, L.A. Peluso, and L.N. Brewer: A diffusionmultiple approach for mapping phase diagrams, hardness, and elastic modulus. JOM 54, 42 (2002).

21. C.A. Schuh and A.C. Lund: Application of nucleation theory to the rate dependence of incipient plasticity during nanoindentation. J. Mater. Res. 19, 2152 (2004).

22. C.A. Schuh, J.K. Mason, and A.C. Lund: Quantitative insight into dislocation nucleation from high-temperature nanoindentation experiments. Nat. Mater. 4, 617 (2005).

23. C.E. Packard, E.R. Homer, N. Al-Aqeeli, and C.A. Schuh: Cyclic hardening of metallic glasses under Hertzian contacts: Experiments and STZ dynamics simulations. Philos. Mag. 90, 1373 (2010).

24. C.E. Packard, L.M. Witmer, and C.A. Schuh: Hardening of a metallic glass during cyclic loading in the elastic range. Appl. Phys. Lett. 92, 171911 (2008).

25. A.C. Fischer-Cripps: Introduction to Contact Mechanics (Springer, New York, 2000).

26. H. Hertz: Miscellaneous Papers, translated by D.E. Jones and G.A. Schott (Macmillan, London, 1896).

27. P.C. Wo, L. Zuo, and A.H.W. Ngan: Time-dependent incipient plasticity in $\mathrm{Ni}_{3} \mathrm{Al}$ as observed in nanoindentation. J. Mater. Res. 20, 489 (2005).

28. K.V. Rajulapati, M.M. Biener, J. Biener, and A.M. Hodge: Temperature dependence of the plastic flow behavior of tantalum. Philos. Mag. Lett. 90, 35 (2010).
29. W.W. Gerberich, J.C. Nelson, E.T. Lilleodden, P. Anderson, and J.T. Wyrobek: Indentation induced dislocation nucleation: The initial yield point. Acta Mater. 44, 3585 (1996).

30. M.M. Biener, J. Biener, A.M. Hodge, and A.V. Hamza: Dislocation nucleation in bcc Ta single crystals studied by nanoindentation. Phys. Rev. B 76, 165422 (2007).

31. C.A. Schuh and T.G. Nieh: A survey of instrumented indentation studies on metallic glasses. J. Mater. Res. 19, 46 (2004).

32. K. Durst, B. Backes, O. Franke, and M. Goken: Indentation size effect in metallic materials: Modeling strength from pop-in to macroscopic hardness using geometrically necessary dislocations. Acta Mater. 54, 2547 (2006).

33. E.K. Njeim and D.F. Bahr: Atomistic simulations of nanoindentation in the presence of vacancies. Scr. Mater. 62, 598 (2010).

34. S. Shim, H. Bei, M.K. Miller, G.M. Pharr, and E.P. George: Effects of focused-ion-beam milling on the compressive behavior of directionally solidified micropillars and the nanoindentation response of an electropolished surface. Acta Mater. 57, 503 (2009).

35. B. Moser, J. Kuebler, H. Meinhard, W. Muster, and J. Michler: Observation of instabilities during plastic deformation by insitu SEM indentation experiments. Adv. Eng. Mater. 7, 388 (2005).

36. B.G. Yoo, J.Y. Kim, and J.I. Jang: Influence of indenter geometry on the deformation behavior of $\mathrm{Zr}_{60} \mathrm{Cu}_{30} \mathrm{Al}_{10}$ bulk metallic glass during nanoindentation. Mater. Trans. 48, 1765 (2007).

37. C.A. Schuh and T.G. Nieh: A nanoindentation study of serrated flow in bulk metallic glasses. Acta Mater. 51, 87 (2003).

38. L. Wang, S.X. Song, and T.G. Nieh: Assessing plastic shear resistance of bulk metallic glasses under nanoindentation. Appl. Phys. Lett. 92, 3 (2008).

39. B. Yang and T.G. Nieh: Effect of the nanoindentation rate on the shear band formation in an Au-based bulk metallic glass. Acto Mater. 55, 295 (2007).

40. A.C. Fischer-Cripps: Nanoindentation (Springer, New York, 2002)

41. C.A. Schuh, T.G. Nieh, and Y. Kawamura: Rate dependence of serrated flow during nanoindentation of a bulk metallic glass J. Mater. Res. 17, 1651 (2002).

42. N. Li, L. Liu, Q. Chen, J. Pan, and K.C. Chan: The effect of free volume on the deformation behaviour of a $\mathrm{Zr}$-based metallic glass under nanoindentation. J. Phys. D: Appl. Phys. 40, 6055 (2007).

43. Y. Liu, T. Zhang, B. Wei, D. Xing, W. Li, and L. Zhang: Effect of structural relaxation on deformation behaviour of Zr-based metallic glass. Chin. Phys. Lett. 23, 1868 (2006).

44. B. Yang, J. Wadsworth, and T.G. Nieh: Thermal activation in Aubased bulk metallic glass characterized by high-temperature nanoindentation. Appl. Phys. Lett. 90, 061911 (2007).

45. K. Saksl, H. Franz, P. Jóvári, K. Klementiev, E. Welther, A. Ehnes, J. Saida, A. Inoue, and J.Z. Jiang: Evidence of icosahedral short-range order in $\mathrm{Zr}_{70} \mathrm{Cu}_{30}$ and $\mathrm{Zr}_{70} \mathrm{Cu}_{29} \mathrm{Pd}_{1}$ metallic glasses. Appl. Phys. Lett. 83, 3924 (2003).

46. J. Sietsma and B.J. Thijsse: An investigation of universal medium range order in metallic glasses. J. Non-Cryst. Solids 135, 146 (1991).

47. D.B. Miracle, T. Egami, K.M. Flores, and K.F. Kelton: Structural aspects of metallic glasses. MRS Bull. 32, 629 (2007).

48. K.M. Flores, E. Sherer, A. Bharathula, H. Chen, and Y.C. Jean: Sub-nanometer open volume regions in a bulk metallic glass investigated by positron annihilation. Acta Mater. 55, 3403 (2007).

49. K.M. Flores, B.P. Kanungo, S.C. Glade, and P. Asoka-Kumar: Characterization of plasticity-induced structural changes in a 
Zr-based bulk metallic glass using positron annihilation spectroscopy. J. Non-Cryst. Solids 353, 1201 (2007).

50. A.S. Argon: Plastic-deformation in metallic glasses. Acta Metall. 27, 47 (1979).

51. F. Spaepen: Microscopic mechanism for steady-state inhomogeneous flow in metallic glasses. Acta Metall. Mater. 25, 407 (1977).

52. J.S. Langer: Shear-transformation-zone theory of deformation in metallic glasses. Scr. Mater. 54, 375 (2006).

53. A.S. Argon and H.Y. Kuo: Free-energy spectra for inelastic deformation of 5 metallic-glass alloys. J. Non-Cryst. Solids 37, 241 (1980).

54. D. Rodney and C. Schuh: Distribution of thermally activated plastic events in a flowing glass. Phys. Rev. Lett. 102, 235503 (2009).

55. C.A. Schuh, A.C. Lund, and T.G. Nieh: New regime of homogeneous flow in the deformation map of metallic glasses: Elevated temperature nanoindentation experiments and mechanistic modeling. Acta Mater. 52, 5879 (2004)

56. D. Klaumunzer, R. Maass, F.H. Dalla Torre, and J.F. Loffler: Temperature-dependent shear band dynamics in a Zr-based bulk metallic glass. Appl. Phys. Lett. 96, 061901 (2010).

57. C.A. Schuh, T.C. Hufnagel, and U. Ramamurty: Mechanical behavior of amorphous alloys. Acta Mater. 55, 4067 (2007).

58. C.E. Packard, J. Schroers, and C.A. Schuh: In situ measurements of surface tension-driven shape recovery in a metallic glass. Scr. Mater. 60, 1145 (2009).

59. N. Nishiyama, A. Inoue, and J.Z. Jiang: Elastic properties of $\mathrm{Pd}_{40} \mathrm{Cu}_{30} \mathrm{Ni}_{10} \mathrm{P}_{20}$ bulk glass in supercooled liquid region. Appl. Phys. Lett. 78, 1985 (2001)

60. W.L. Johnson and K. Samwer: A universal criterion for plastic yielding of metallic glasses with a $\left(T / T_{g}\right)^{2 / 3}$ temperature dependence. Phys. Rev. Lett. 95, 195501 (2005).

61. E.R. Homer, D. Rodney, and C.A. Schuh: Kinetic Monte Carlo study of activated states and correlated shear-transformation-zone activity during the deformation of an amorphous metal. Phys. Rev. B 81, 064204 (2010).

62. E.R. Homer and C.A. Schuh: Three-dimensional shear transformation zone dynamics model for amorphous metals. Modell. Simul. Mater. Sci. Eng. 18, 065009 (2010).

63. A. Slipenyuk and J. Eckert: Correlation between enthalpy change and free volume reduction during structural relaxation of $\mathrm{Zr}_{55} \mathrm{Cu}_{30} \mathrm{Al}_{10} \mathrm{Ni}_{5}$ metallic glass. Scr. Mater. 50, 39 (2004).

64. A. Vandenbeukel and J. Sietsma: The glass-transition as a freevolume related kinetic phenomenon. Acta Metall. Mater. 38, 383 (1990).

65. U. Ramamurty, M.L. Lee, J. Basu, and Y. Li: Embrittlement of a bulk metallic glass due to low-temperature annealing. Scr. Mater. 47, 107 (2002).

66. J.J. Lewandowski, W.H. Wang, and A.L. Greer: Intrinsic plasticity or brittleness of metallic glasses. Philos. Mag. Lett. 85, 77 (2005).

67. T. Mukai, T.G. Nieh, Y. Kawamura, A. Inoue, and K. Higashi: Dynamic response of a $\mathrm{Pd}_{40} \mathrm{Ni}_{40} \mathrm{P}_{20}$ bulk metallic glass in tension. Scr. Mater. 46, 43 (2002).

68. X. Hu, S.C. Ng, Y.P. Feng, and Y. Li: Glass forming ability and in-situ composite formation in Pd-based bulk metallic glasses. Acta Mater. 51, 561 (2003).

69. J. Shen, Q.J. Chen, J.F. Sun, H.B. Fan, and G. Wang: Exceptionally high glass-forming ability of an $\mathrm{FeCoCrMoCBY}$ alloy. Appl. Phys. Lett. 86, 151907 (2005).

70. J.T. Trenkle, C.E. Packard, and C.A. Schuh: Hot nanoindentation in inert environments. Rev. Sci. Instrum. 81, 073901 (2010).

71. C.A. Schuh, C.E. Packard, and A.C. Lund: Nanoindentation and contact-mode imaging at high temperatures. J. Mater. Res. 21, 725 (2006).

72. Z.C. Duan and A.M. Hodge: High-temperature nanoindentation: New developments and ongoing challenges. JOM 61, 32 (2009).

\section{APPENDIX}

In this Appendix we assemble the various experimental test conditions and material data used in the body of the paper. All of the room-temperature nanoindentation experiments reported in the paper were conducted using a Hysitron nanoindenter. Room-temperature test procedures used for each specimen were:

(i) (110) Oriented Single-Crystal Platinum. The data in this paper are reproduced from the prior work of Mason and coworkers. ${ }^{4,22}$ The diamond tip radius used for this sample was $150 \mathrm{~nm}$, and the sample surface was mechanically and then electrochemically polished. The reduced modulus of $\mathrm{Pt}$ is $171 \mathrm{GPa}$.

(ii) $\mathrm{Pd}_{40} \mathrm{Ni}_{40} \mathrm{P}_{20}$ Metallic Glass. The NSD data for this material, tested with a conospherical tip of radius $590 \mathrm{~nm}$, are reported here for the first time, although some of the experiments (including surface preparation) are described in Ref. 9. Material synthesis details are available in Ref. 67. The reduced modulus of the specimen is $117 \mathrm{GPa}$. The baseline rate for testing was $0.2 \mathrm{mN} / \mathrm{s}$, and the data in Fig. 7 also show data acquired at lower rates of 0.02 and $0.002 \mathrm{mN} / \mathrm{s}$.

(iii) $\mathrm{Pd}_{40} \mathrm{Cu}_{30} \mathrm{Ni}_{10} \mathrm{P}_{20}$ Metallic Glass. The data for this material with a tip radius of $1.2 \mu \mathrm{m}$ are new to the present paper, although some different tests, using a different tip radius $(1.1 \mu \mathrm{m})$ and studying different phenomena, are reported in Ref. 23. That reference also contains details on the sample preparation procedures. Material synthesis is described in Ref. 68 and elastic properties in Ref. 59. At room temperature the reduced modulus of this material is $100 \mathrm{GPa}$. The rate used in the experiments was $0.5 \mathrm{mN} / \mathrm{s}$.

(iv) $\mathrm{Fe}_{41} \mathrm{Co}_{7} \mathrm{Cr}_{15} \mathrm{Mo}_{14} \mathrm{C}_{15} \mathrm{~B}_{6} \mathrm{Y}_{2}$ Metallic Glass. Some of the data for this material appeared previously in Refs. 23 and 24, where details on surface preparation are also provided. Material preparation details can be found in Ref. 69. Additional new data for this material are also presented, using a $1.1 \mu \mathrm{m}$ radius tip, and a reduced modulus of $217 \mathrm{GPa}$. The baseline rate used to acquire the NSD was $0.3 \mathrm{mN} / \mathrm{s}$, although additional data are presented using rates covering the range 0.2 to 25 $\mathrm{mN} / \mathrm{s}$, with a peak load of $4 \mathrm{mN}$.

In addition to the room-temperature experiments described above, additional new experiments were performed involving nonambient temperatures. The data shown in Fig. 11 are for glasses that were exposed to a $12 \mathrm{~h}$ anneal at $80 \%$ their respective glass-transition temperatures $\left(T_{\mathrm{g}}=303\right.$ and $565{ }^{\circ} \mathrm{C}$ for $\mathrm{Pd}_{40} \mathrm{Ni}_{40} \mathrm{P}_{20}$ and $\mathrm{Fe}_{41} \mathrm{Co}_{7} \mathrm{Cr}_{15} \mathrm{Mo}_{14} \mathrm{C}_{15} \mathrm{~B}_{6} \mathrm{Y}_{2}$, respectively). High-temperature nanoindentation experiments were conducted on the $\mathrm{Pd}_{40} \mathrm{Cu}_{30} \mathrm{Ni}_{10} \mathrm{P}_{20}$ glass, using a custom apparatus described in Ref. 70, which not only incorporates a heating stage, but also a controlled-atmosphere chamber that permits testing in an inert environment. Experiments 
were conducted at room temperature $\left(24^{\circ} \mathrm{C}\right), 50,100$, and $150{ }^{\circ} \mathrm{C}\left(0.52,0.57,0.66\right.$, and $0.75 T_{\mathrm{g}}$, respectively), with a loading rate of $500 \mu \mathrm{N} / \mathrm{s}$ and a diamond tip radius of $1.2 \mu \mathrm{m}$. At the four test temperatures, the reduced moduli are $E_{\mathrm{r}} \approx 100,98,97$, and $95 \mathrm{GPa}$, respectively. A peak load of $3 \mathrm{mN}$ was used at room temperature, but this was lowered to $2 \mathrm{mN}$ at elevated temperatures. The system was equilibrated for $45 \mathrm{~min}$ prior to testing at elevated temperatures, and the tip was kept in contact with the surface throughout the experiments in accordance with the procedure described in Refs. 70 to 72 . In order to avoid oxidation at nonambient temperatures, the tests were performed in a controlled atmosphere attained by drawing a mechanical vacuum and backfilling with ultrahigh-purity argon three times. The final base pressure was $<2.5 \mathrm{~Pa}$ during testing. A series of room-temperature reference measurements were repeated twice (after the tests at 50 to $100{ }^{\circ} \mathrm{C}$ and also after those at higher temperatures); through this procedure we verified that the room-temperature NSD was not affected by high-temperature testing (due to either oxidation or other structural change).

The simulations described in the manuscript are based on the STZ dynamics simulation method described in detail in Ref. 12, and its adaptation to the case of indentation contact in Ref. 23; the results reported here, however, are all new to the present work. The simulated glass was $0.7 \mathrm{~nm}$ thick (plane strain conditions were applied in this direction), $100 \mathrm{~nm}$ wide, and $35 \mathrm{~nm}$ high. The input parameters that describe the glass properties included a shear modulus of $35.8 \mathrm{GPa}$, Poisson's ratio of 0.352 , Debye temperature of $327 \mathrm{~K}$, an STZ volume of $1.6 \mathrm{~nm}^{3}$, and an intrinsic activation energy barrier of $2.63 \mathrm{eV}$, as taken from Refs. 12, 23 and 61. Prior to testing the glass, an internal stress/strain distribution was developed as follows. The pre-existing stress distribution for this glass was developed through simulated thermal processing in the STZ dynamics framework by allowing a simulation cell to reach an equilibrated state through STZ activity at $1000 \mathrm{~K}$ and then allowing the glass to relax through STZ activity during cooling of the glass at $10 \mathrm{~K} / \mathrm{s}$ until it reached $300 \mathrm{~K}$, as described in Ref. 12. This pre-existing stress distribution was then mapped onto the present simulation cell and allowed a minor degree of equilibration to satisfy the boundary conditions used in the present simulations. These conditions (which were used for the indentation simulations) included fixed nodes along the bottom edge of the cell, and periodic boundary conditions at the sides of the cell. Frictionless hard contact was assumed between the tip and the top of the simulation cell, which was otherwise free. The tip radius was $40 \mathrm{~nm}$ and the elastic reference curve in Fig. 9 was obtained by suppressing STZ activity to recover the purely elastic response of the model. 